\title{
Impact of Prior Ischemic Stroke on Outcomes in Patients With Heart Failure
}

\author{
- A Propensity-Matched Study -
}

\author{
Yu Wang, MD; Meng-Xi Yang, MD; Qiang Tu, PhD; Li-Yuan Tao, MD; \\ Gang Liu, MD; Hui An, MD; Hu Zhang, MD; Jiang-Li Jin, MD; \\ Jia-Sai Fan, MD; Yi-Fei Du, MD; Jin-Gang Zheng, MD; Jing-Yi Ren, MD
}

\begin{abstract}
Background: Whether ischemic stroke per se, rather than older age or additional comorbidities, accounts for the adverse prognosis of heart failure (HF) is uncertain. The present study examineed the intrinsic association of ischemic stroke with outcomes in a propensity-matched cohort.
\end{abstract}

Methods and Results: Of 1,351 patients hospitalized with HF, $388(28.7 \%)$ had prior ischemic stroke. Using propensity score for prior ischemic stroke, estimated for each patient, a matched cohort of 379 pairs of HF patients with and without prior ischemic stroke, balanced on 32 baseline characteristics was assembled. At 30 days, prior ischemic stroke was associated with significantly higher risks of the combined endpoint of all-cause death or readmission (hazard ratio [HR]: 1.91; 95\% confidence interval [Cl]: 1.38 to 2.65 ; $\mathrm{P}<0.001$ ), all-cause death (HR: $2.08 ; 95 \% \mathrm{Cl}: 1.28$ to 3.38 ; $\mathrm{P}=0.003$ ), all-cause readmission (HR: $2.67 ; 95 \% \mathrm{Cl}$ : 1.78 to 4.01 ; $\mathrm{P}<0.001$ ), and $\mathrm{HF}$ readmission (HR: $2.11 ; 95 \% \mathrm{Cl}: 1.19$ to $3.72 ; \mathrm{P}=0.010$ ). Prior ischemic stroke was associated with a significantly higher risk of all 4 outcomes at both 6 months and 1 year.

Conclusions: Prior ischemic stroke was a potent and persistent risk predictor of death and readmission among patients with HF after accounting for clinical characteristics.

Key Words: Death; Heart failure; Ischemic stroke; Propensity score; Readmission

$\mathbf{H}$ eart failure (HF) is predominantly a complex disease of older adults with extensive comorbidities that have substantial implications for prognosis. ${ }^{1-3}$ Stroke, especially ischemic stroke responsible for the majority of strokes, is a common comorbidity in patients with HF.,5 There is a general perception that the interaction between ischemic stroke and HF deteriorates the outcomes of HF because the 2 diseases share common risk factors and pathophysiological mechanisms. ${ }^{6}$

To date, there is a paucity of studies investigating the effect of prior ischemic stroke on outcomes in real-world patients with $\mathrm{HF}$, and several large cohorts failed to identify stroke as a significant predictor of $\mathrm{HF}$ prognosis in
Western populations..$^{7-9}$ Associations between stroke and poor outcomes of HF have often been concealed by the bias of older age and higher prevalence of additional comorbidities that affected HF prognosis. Further, the bias is unable to be adjusted using traditional regression-based multivariable risk models because of the limitation of lack of procedural transparency, confounders that may not be balanced at baseline, and strong and often untenable model assumptions. ${ }^{10}$ Accordingly, whether stroke per se, rather than age or comorbidities, accounts for adverse prognosis is uncertain. Propensity score matching, on the other hand, is designed to assemble cohorts in which patients are balanced on all measured baseline covariates

Received March 13, 2020; revised manuscript received June 26, 2020; accepted July 6, 2020; J-STAGE Advance Publication released online September 4, 2020 Time for primary review: 18 days

Department of Cardiology, China-Japan Friendship Hospital, Beijing (Y.W., M.-X.Y., H.Z., J.-S.F., Y.-F.D., J.-G.Z., J.-Y.R.); State Key Laboratory for Molecular and Developmental Biology, Institute of Genetics and Developmental Biology, Chinese Academy of Sciences, Beijing (Q.T.); University of Chinese Academy of Sciences, Beijing (Q.T.); Research Center of Clinical Epidemiology, Peking University Third Hospital, Beijing (L.-Y.T.); Department of Cardiovascular Surgery, Peking University People's Hospital, Beijing (G.L.); Department of Cardiology, Hebei General Hospital, Hebei (H.A.); Department of Neurology, China-Japan Friendship Hospital, Beijing (J.-L.J.); and Vascular Health Research Center of Peking University Health Science Center, Beijing (J.-Y.R.), China

The first two authors contributed equally to this work (Y.W., M.-X.Y.).

Mailing address: Jingyi Ren, MD, Department of Cardiology, China-Japan Friendship Hospital, 2 Yinghua Dongjie, Hepingli, Beijing 100029, China. E-mail: renjingyi1213@hotmail.com

All rights are reserved to the Japanese Circulation Society. For permissions, please e-mail: cj@j-circ.or.jp

ISSN-1346-9843 
Patients enrolled from January 1, 2015, to January 30, $2018(n=1458)$

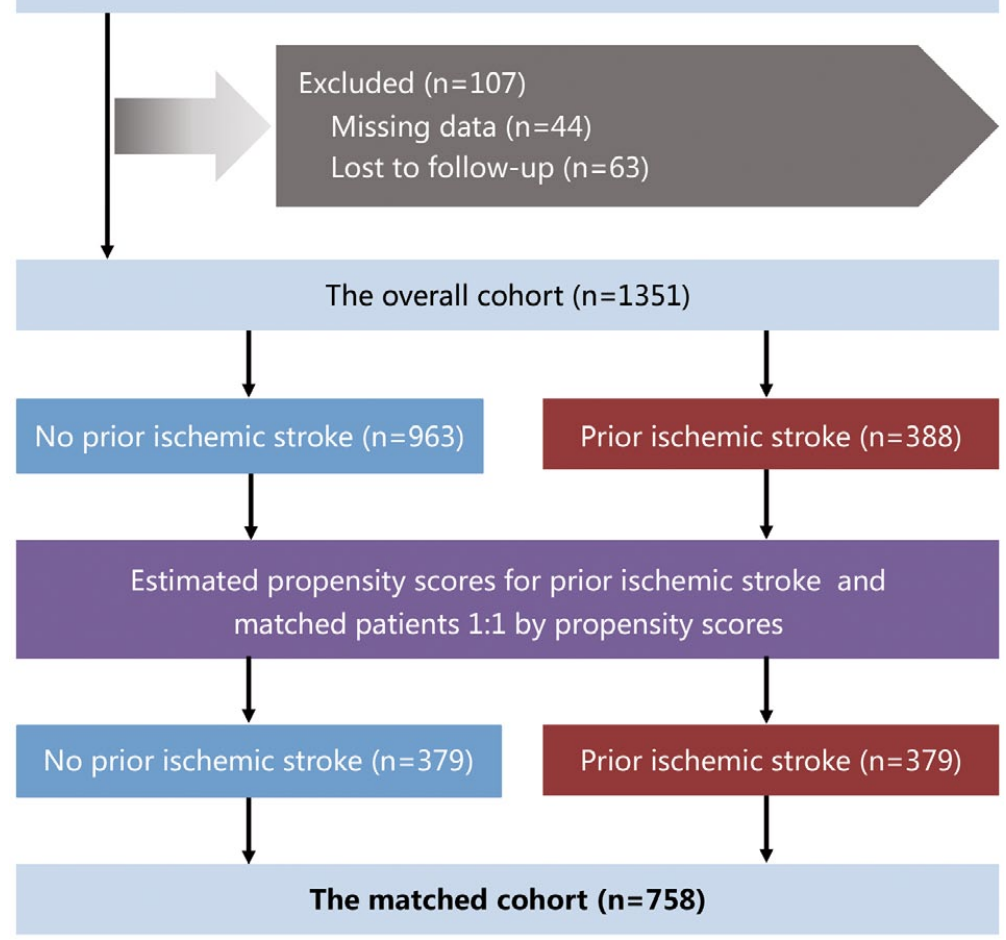

Figure 1. Flow chart of the assembly of a matched cohort of patients with heart failure, according to prior ischemic stroke. and investigators are blinded to study outcomes. ${ }^{11-14}$ The use of propensity scores to perform adjustment also allows investigators to assess the extent to which stroke has an independent association with poor outcomes.

Therefore, the objective of the present study was to examine the association of prior ischemic stroke with outcomes in a propensity score-matched cohort of patients with HF in which those with and without stroke would be well balanced on all measured baseline covariates. An understanding of this association could have important implications for future prevention strategies in these particular patients with $\mathrm{HF}$.

\section{Methods}

\section{Study Population}

This was a prospective observational cohort study of patients hospitalized in the Heart Failure Center of ChinaJapan Friendship Hospital between January 1, 2015, and January 30, 2018. All patients aged 18 years or older admitted to the department with a diagnosis of HF were enrolled. Patients with HF were identified by the clinical practice guideline of the Chinese Society of Cardiology, with diagnostic criteria that are similar to the recommendations of the European Society of Cardiology. ${ }^{15,16}$ Cases of missing data or lost to follow-up were excluded. The patient selection process is shown in Figure 1. The Ethics Committee of the China-Japan Friendship Hospital approved the overall protocol.

\section{Definition of Prior Ischemic Stroke}

Prior ischemic stroke was clinically ascertained according to the guidelines of the American Heart Association/American Stroke Association with minor modification because patients had the ischemic stroke 6-60 months prior, with stable neuropathological, neuroimaging, and/or clinical evidence of permanent injury. ${ }^{17}$ In 388 patients, prior ischemic stroke was diagnosed.

\section{Data Collection}

Variables collected in the present study included demographics (age, sex, and history of smoking), echocardiographic and biochemical data (albumin, B-type natriuretic peptide, blood urea nitrogen, etc.), the etiology of HF (ischemic heart disease, cardiomyopathy, etc.), comorbidities (cardiac and non-cardiac) at admission, and medications (angiotensin-converting enzyme inhibitors [ACEIs] or angiotensin-receptor blockers [ARBs], $\beta$-blockers, mineralocorticoid receptor antagonists [MRAs], calciumchannel blockers [CCBs], statins, diuretics, nitrates, digoxin, antiplatelets, and anticoagulants) at admission and discharge.

\section{Assembly of a Balanced Cohort}

First, we analyzed the factors related to 30-day all-cause readmission or death in the 388 hospitalized HF patients with prior ischemic stroke. Briefly, in the univariable analysis for 30-day all-cause readmission or death, age, DBP, N-terminal pro-B-type natriuretic peptide (NTproBNP), hemoglobin, total hospital length of stay, whitecell count, neutrophil-to-lymphocyte ratio serum albumin, serum sodium, serum potassium, triglycerides, thyroid stimulating hormone, triiodothyronine, anemia, dyslipidemia, infection, thyroid disease, ACEIs/ARBs, $\beta$-blockers, 


\begin{tabular}{|c|c|c|c|c|c|c|}
\hline \multirow[b]{2}{*}{ Variable } & \multicolumn{3}{|c|}{ Entire cohort } & \multicolumn{3}{|c|}{ Propensity score-matched cohort } \\
\hline & $\begin{array}{c}\text { No prior ischemic } \\
\text { stroke }(n=963)\end{array}$ & $\begin{array}{l}\text { Prior ischemic } \\
\text { stroke }(n=388)\end{array}$ & $P$ value & $\begin{array}{l}\text { No prior ischemic } \\
\text { stroke }(n=379)\end{array}$ & $\begin{array}{l}\text { Prior ischemic } \\
\text { stroke }(n=379)\end{array}$ & $P$ value \\
\hline Age, years & $66.7 \pm 14.3$ & $75.6 \pm 10.1$ & $<0.001$ & $75.0 \pm 9.5$ & $77.6 \pm 16.2$ & 0.508 \\
\hline Female & $372(38.6)$ & $161(41.5)$ & 0.330 & $211(55.7)$ & $220(58.0)$ & 0.509 \\
\hline $\mathrm{SBP}, \mathrm{mmHg}$ & $129.0 \pm 22.0$ & $130.7 \pm 24.4$ & 0.218 & $133.4 \pm 24.5$ & $131.1 \pm 23.9$ & 0.190 \\
\hline $\mathrm{DBP}, \mathrm{mmHg}$ & $74.9 \pm 13.2$ & $74.5 \pm 14.8$ & 0.600 & $73.6 \pm 13.8$ & $74.5 \pm 14.5$ & 0.747 \\
\hline Heart rate, beats/min & $79.1 \pm 15.9$ & $77.8 \pm 16.2$ & 0.155 & $78.3 \pm 16.7$ & $77.6 \pm 16.2$ & 0.552 \\
\hline $\mathrm{BMI}, \mathbf{k g} / \mathrm{m}^{2}$ & $24.7 \pm 5.8$ & $24.8 \pm 3.0$ & 0.594 & $24.7 \pm 4.5$ & $24.90 \pm 3.9$ & 0.589 \\
\hline History of smoking & $453(47.0)$ & $147(37.9)$ & 0.088 & $133(35.1)$ & $140(36.9)$ & 0.864 \\
\hline \multicolumn{7}{|l|}{ Past medical history } \\
\hline CAD & $607(63.0)$ & $282(72.7)$ & 0.001 & $260(68.6)$ & $273(72.0)$ & 0.301 \\
\hline Hypertension & $687(71.3)$ & $322(83.0)$ & $<0.001$ & $314(82.8)$ & $316(83.4)$ & 0.846 \\
\hline Dyslipidemia & $451(46.8)$ & $222(57.2)$ & 0.001 & $229(60.4)$ & $220(58.0)$ & 0.506 \\
\hline Diabetes mellitus & $351(36.4)$ & $166(42.8)$ & 0.031 & $161(42.5)$ & $164(43.3)$ & 0.760 \\
\hline Atrial fibrillation & $302(31.4)$ & $145(37.4)$ & 0.035 & $139(36.7)$ & $138(36.4)$ & 0.940 \\
\hline Anemia & $309(32.1)$ & $153(39.4)$ & 0.010 & $152(40.1)$ & $146(38.5)$ & 0.655 \\
\hline CKD & $277(28.8)$ & $101(26.0)$ & 0.309 & $87(23.0)$ & $100(26.4)$ & 0.273 \\
\hline Cancer & $176(18.3)$ & $92(23.7)$ & 0.025 & $86(22.7)$ & $86(22.7)$ & 1.000 \\
\hline COPD & $170(17.7)$ & $47(12.1)$ & 0.010 & $44(11.6)$ & $46(12.1)$ & 0.822 \\
\hline Thyroid dysfunction & $52(5.4)$ & $27(7.0)$ & 0.277 & $25(6.6)$ & $27(7.1)$ & 0.774 \\
\hline \multicolumn{7}{|l|}{ Laboratory findings } \\
\hline Hemoglobin, g/L & $125.3 \pm 24.5$ & $119.6 \pm 21.3$ & $<0.001$ & $118.6 \pm 23.3$ & $119.9 \pm 21.3$ & 0.431 \\
\hline eGFR, $\mathrm{mL} / \mathrm{min} / 1.73 \mathrm{~m}^{2}$ & $76.3 \pm 30.6$ & $66.9 \pm 28.4$ & $<0.001$ & $66.4 \pm 29.6$ & $67.1 \pm 28.3$ & 0.756 \\
\hline Glucose, $\mathrm{mmol} / \mathrm{L}$ & $7.2 \pm 3.1$ & $7.7 \pm 3.5$ & 0.023 & $7.6 \pm 3.4$ & $7.62 \pm 3.5$ & 0.805 \\
\hline $\mathrm{BNP}, \mathrm{pg} / \mathrm{mL}^{*}$ & $\begin{array}{c}580.5 \\
(366.0,1,064.0)\end{array}$ & $\begin{array}{c}485.5 \\
(267.5,843.0)\end{array}$ & $<0.001$ & $\begin{array}{c}530.0 \\
(281.0,933.0)\end{array}$ & $\begin{array}{c}494.0 \\
(267.0,847.0)\end{array}$ & 0.319 \\
\hline LVEF, \% & $51.6 \pm 12.9$ & $53.54 \pm 9.6$ & 0.002 & $50.97 \pm 8.5$ & $51.38 \pm 7.0$ & 0.463 \\
\hline \multicolumn{7}{|l|}{ Medications at admission } \\
\hline ACEIs/ARBs & $633(65.7)$ & $262(67.5)$ & 0.527 & $252(66.5)$ & $251(66.2)$ & 0.939 \\
\hline$\beta$-blockers & $663(68.8)$ & $256(66.0)$ & 0.308 & $263(69.4)$ & $256(67.5)$ & 0.584 \\
\hline MRAs & $402(41.7)$ & $139(35.8)$ & 0.044 & $138(36.4)$ & $138(36.4)$ & 1.000 \\
\hline Statins & $374(38.8)$ & $169(43.6)$ & 0.110 & $164(43.3)$ & $168(44.3)$ & 0.770 \\
\hline Loop diuretics & 789 (81.9) & $335(86.3)$ & 0.046 & $328(86.5)$ & $335(88.4)$ & 0.442 \\
\hline Nitrates & $485(50.4)$ & $210(54.1)$ & 0.211 & $193(50.9)$ & $201(53.0)$ & 0.561 \\
\hline Digoxin & $254(26.4)$ & $79(20.4)$ & 0.019 & $80(21.1)$ & $79(20.8)$ & 0.929 \\
\hline Antiplatelet drugs & $507(52.6)$ & $226(58.2)$ & 0.061 & $210(55.4)$ & $223(58.8)$ & 0.340 \\
\hline Anticoagulants & $213(22.1)$ & $52(13.4)$ & $<0.001$ & $55(14.5)$ & $47(12.4)$ & 0.394 \\
\hline CCBs & $279(29.0)$ & $139(35.8)$ & 0.014 & $130(34.3)$ & $139(36.7)$ & 0.494 \\
\hline
\end{tabular}

Values are mean \pm standard deviation or number (\%) unless indicated otherwise. ${ }^{*}$ Median [interquartile range]. ACEI, angiotensin-converting enzyme inhibitor; ARB, angiotensin-receptor blocker; BMI, body mass index; BNP, B-type natriuretic peptide; CAD, coronary artery disease; $\mathrm{CCB}$, calcium-channel blocker; CKD, chronic kidney disease; COPD, chronic obstructive pulmonary disease; DBP, diastolic blood pressure; eGFR, estimated glomerular filtration rate; LVEF, left ventricular ejection fraction; MRA, mineralocorticoid receptor antagonist; SBP, systolic blood pressure.

nitrates, antiplatelets, statins and CCBs (all $\mathrm{P}<0.05)$ (Supplementary Table 1).

Due to the imbalance in baseline covariates and additional covariates that affected HF prognosis, propensity score matching was used to assemble a cohort in which patients with and without prior ischemic stroke would be balanced on key measured baseline covariates. A notable strength of using the propensity score approach is that the process of cohort assembly is outcome-blinded as in a randomized controlled trial, and the balanced baseline characteristics can be displayed in a tabular form. ${ }^{11,12} \mathrm{We}$ estimated the propensity score for prior ischemic stroke for each of 1,351 patients using a non-parsimonious multivariable logistic regression model. ${ }^{\mathbf{1 8 - 2 0}}$ In the model, prior ischemic stroke was the dependent variable and 32 baseline characteristics (Table 1) were the covariates. Using a matching protocol, we matched 379 , or $97.7 \%$, of the 388 patients who had prior ischemic stroke, with another 379 patients without prior ischemic stroke, but with a similar probability or propensity score as the patients with prior ischemic stroke. The distribution of propensity scores is shown in Figure 2. As propensity score models are samplespecific adjusters and are not intended to be utilized for out-of-sample prediction or estimation of coefficients, the measures of fitness and discrimination are not important for the assessment of the model's effectiveness. ${ }^{21}$ Hence, to quantify pre-match imbalances and post-match balances, we assessed our propensity score model by calculating the 


\section{A}

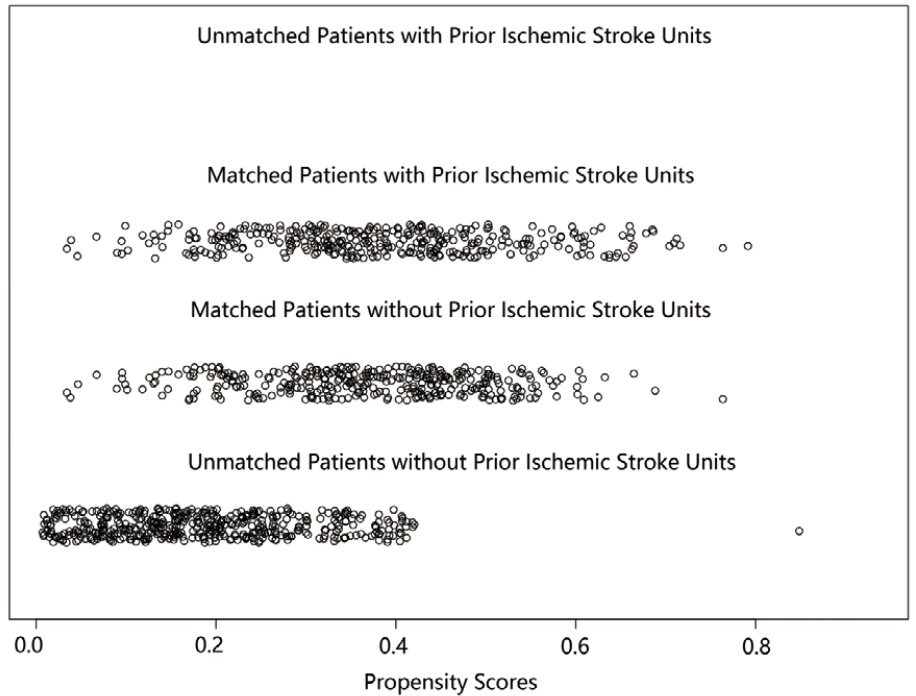

B

Raw Patients with Prior Ischemic Stroke

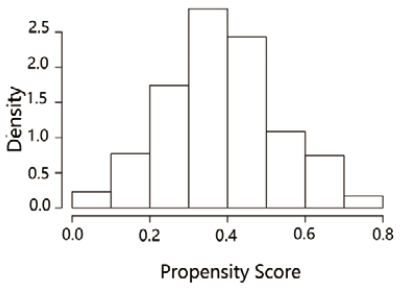

Raw Patients without Prior Ischemic Stroke

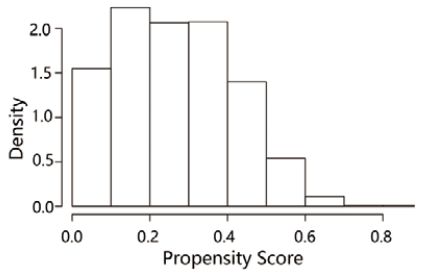

Matched Patients with Prior Ischemic Stroke
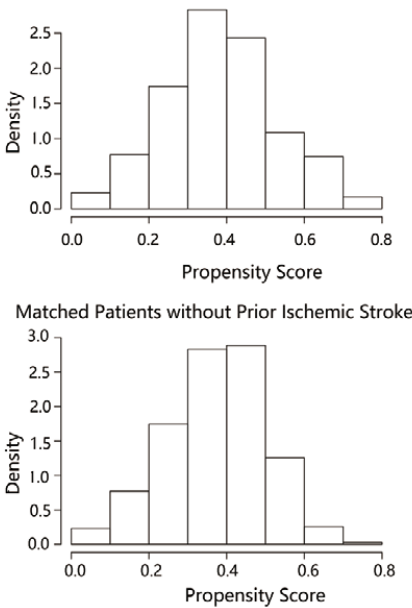

Figure 2. Results of propensity score-matched, by prior ischemic stroke. (A) Distribution of propensity scores for the cohort before and after matching, by prior ischemic stroke. (B) Histograms of the density of propensity scores for the cohort before and after matching, by prior ischemic stroke. pre- and post-match absolute standardized differences for the 32 measured baseline characteristics, with an absolute standardized difference value of $0 \%$ indicating no residual bias and values $<10 \%$ suggesting inconsequential bias.

\section{Study Outcomes}

We examined the following outcomes: the combined endpoint of all-cause readmission or death, all-cause death, all-cause readmission, and HF readmission. We performed follow-up interviews regularly and all outcome events of death or readmission at 30 days, 6 months, and 1 year were documented into the China Heart Failure Center Reporting Platform (http://data.chinahfc.org). Only the first readmission occurring at any time after the index admission and throughout the follow-up period of the study was considered to be a qualifying readmission.

\section{Statistical Analysis}

Normally distributed continuous variables are expressed as mean \pm standard deviation, non-normally distributed continuous variables are expressed as median and interquartile range, and categorical variables are expressed as n (\%). Pearson chi-square and Wilcoxon rank sum tests were used for the comparisons of baseline characteristics between patients with and without prior ischemic stroke, as appropriate. All outcome analyses were conducted 


\begin{tabular}{|c|c|c|c|c|}
\hline & \multicolumn{2}{|c|}{ Events (\%) } & \multirow[b]{2}{*}{ HR (95\% Cl) } & \multirow[b]{2}{*}{$P$ value } \\
\hline & $\begin{array}{l}\text { No prior ischemic } \\
\text { stroke }(n=379)\end{array}$ & $\begin{array}{l}\text { Prior ischemic } \\
\text { stroke }(n=379)\end{array}$ & & \\
\hline \multicolumn{5}{|l|}{ 30-day outcomes } \\
\hline HF readmission & $18(4.7)$ & $35(9.2)$ & $2.11(1.19-3.72)$ & 0.010 \\
\hline All-cause readmission & $33(8.7)$ & $81(21.4)$ & $2.67(1.78-4.01)$ & $<0.001$ \\
\hline All-cause death & $25(6.6)$ & $48(12.7)$ & $2.08(1.28-3.38)$ & 0.003 \\
\hline All-cause death or readmission & $57(15.0)$ & $100(26.4)$ & $1.91(1.38-2.65)$ & $<0.001$ \\
\hline \multicolumn{5}{|l|}{ 6-month outcomes } \\
\hline HF readmission & $54(14.2)$ & $71(18.7)$ & $1.51(1.06-2.15)$ & 0.023 \\
\hline All-cause readmission & $86(22.7)$ & $147(38.8)$ & $1.95(1.49-2.54)$ & $<0.001$ \\
\hline All-cause death & $38(10.0)$ & $70(18.5)$ & $2.06(1.39-3.05)$ & $<0.001$ \\
\hline All-cause death or readmission & $123(32.5)$ & $174(45.9)$ & $1.60(1.27-2.02)$ & $<0.001$ \\
\hline \multicolumn{5}{|l|}{ 1-year outcomes } \\
\hline HF readmission & $70(18.5)$ & $91(24.0)$ & $1.54(1.13-2.10)$ & 0.007 \\
\hline All-cause readmission & $110(29.0)$ & $183(48.3)$ & $1.95(1.54-2.47)$ & $<0.001$ \\
\hline All-cause death & 39 (10.3) & $86(22.7)$ & $2.51(1.72-2.67)$ & $<0.001$ \\
\hline All-cause death or readmission & $148(39.1)$ & $212(55.9)$ & $1.67(1.35-2.06)$ & $<0.001$ \\
\hline
\end{tabular}

$\mathrm{Cl}$, confidence interval; $\mathrm{HF}$, heart failure; $\mathrm{HR}$, hazard ratio.

using matched data. Cumulative risk for outcomes was estimated by Kaplan-Meier survival curves and compared using the Cox regression analysis. Hazard ratio (HR) and $95 \%$ confidence interval (CI) expressed the risk of events of patients with prior ischemic stroke compared with patients without prior ischemic stroke. Subgroup analyses were performed to determine whether the association between prior ischemic stroke and the combined endpoint of allcause death or readmission was homogeneous across various subgroups of the matched cohort. Two-sided tests with $\mathrm{P}<0.05$ were considered statistically significant. Statistical analysis was conducted using SPSS for Windows (version 25.0, SPSS Inc., Chicago, IL, USA) and the R programming language and environment version 3.6.0 (http://cran.r-project.org).

\section{Results}

\section{Baseline Characteristics of Entire Cohort and Propensity Score-Matched Cohort}

The 758 matched patients had a mean age of $75.3 \pm 9.8$ years and $43.1 \%$ were female. Before matching, 388 (28.7\%) patients had a prior ischemic stroke. Patients with prior ischemic stroke were older, had a greater burden of comorbidities, more HF was caused by coronary artery disease and fewer received MRAs compared with those without prior ischemic stroke (Table 1, Supplementary Table 2). After propensity score matching, all imbalanced baseline characteristics between patients with and without prior ischemic stroke were well balanced so that no significant differences remained (Table 1, Supplementary Table 2). The median (IQR) duration of ischemic stroke of the enrolled patients was 4 years ([3-8] years). The ischemic stroke duration of nearly $80 \%$ patients enrolled in the present study ranged from 3 to 8 years.

\section{0-Day Outcomes}

Prior ischemic stroke was associated with significantly higher risks of the combined endpoint of all-cause death or readmission (HR: 1.91; 95\% $\mathrm{CI}$ : 1.38 to 2.65 ; $\mathrm{P}<0.001$ ), all-cause death (HR: 2.08; 95\% CI:1.28 to $3.38 ; \mathrm{P}=0.003$ ), all-cause readmission (HR: $2.67 ; 95 \% \mathrm{CI}$ : 1.78 to 4.01 ; $\mathrm{P}<0.001$ ), and $\mathrm{HF}$ readmission (HR: $2.11 ; 95 \% \mathrm{CI}: 1.19$ to 3.72; $\mathrm{P}=0.010$ ) (Table 2, Figure 3). The results showed that the duration of ischemic stroke in patients with and without 30 -day readmission or death was $61.1 \pm 34.1$ months and $60.8 \pm 34.7$ months, respectively $(\mathrm{P}=0.291)$. There was no significant difference in the duration of ischemic stroke between the 2 groups, which suggested that stroke per se rather than duration of disease accounted for the poor clinical outcomes in patients with HF.

The major causes of 30-day readmission were non-HF rather than $\mathrm{HF}(56.8 \%$ vs. $43.2 \%)$ in patients with prior ischemic stroke (Supplementary Figure A). The 3 most common non-cardiovascular causes were pneumonia $(32.6 \%)$, renal disorders $(28.3 \%)$, and injury (19.6\%) (Supplementary Figure B).

\section{6-Month and 1-Year Outcomes}

Prior ischemic stroke was associated with higher risks of the combined endpoint of all-cause death or readmission, all-cause death, all-cause readmission, and HF readmission at 6 months and 1 year (Table 2, Figure 3). At 1-year readmission, the proportion of non-HF causes was similar to $\mathrm{HF}$ cause $(24.3 \%$ vs. $24.0 \%)$ in patients with prior ischemic stroke.

\section{Subgroup Analysis in the Matched Cohort}

According to the analyses of outcomes, prior ischemic stroke had a higher weight on adverse outcomes at 30 days compared with 6 months and 1 year. Subgroup analyses for the combined endpoint of 30-day all-cause death or readmission were performed to identify factors that may deteriorate prognosis (Figure 4). The association between prior ischemic stroke and the combined endpoint of allcause death or readmission at 30 days was homogeneous among various clinically relevant subgroups of patients, except by age and statin use at admission. The HRs $(95 \%$ CIs) for the 30-day combined endpoint associated with prior ischemic stroke in the subgroups aged $\geq 75$ years and 

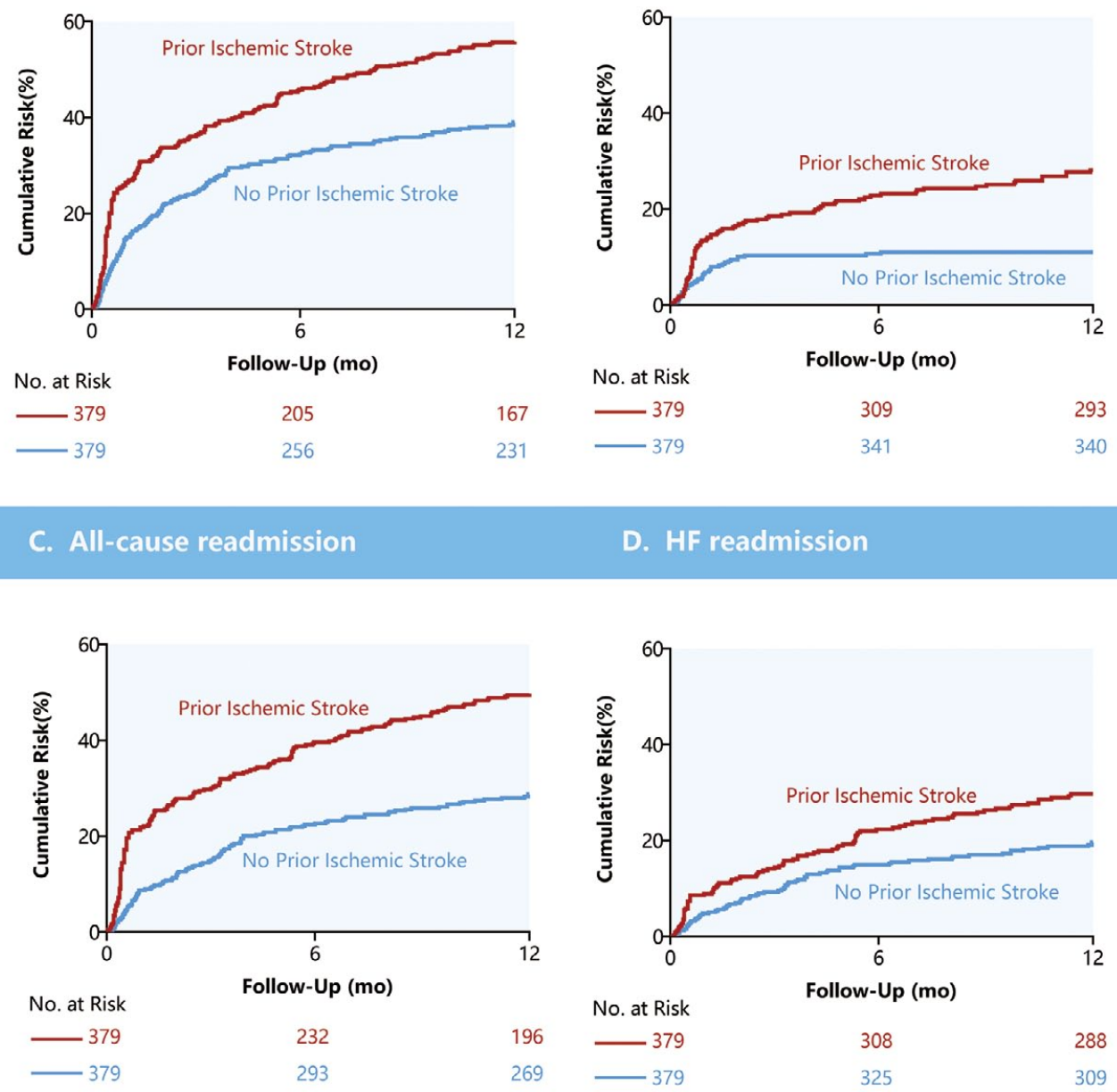

Figure 3. Cumulative risk of outcomes in patients with heart failure (HF), by prior ischemic stroke. Cumulative risk for all-cause death or readmission $(\mathbf{A})$, all-cause death $(\mathbf{B})$, all-cause readmission $(\mathbf{C})$, and HF readmission (D).

$<75$ years were 2.59 (1.71 to $3.93 ; \mathrm{P}<0.001)$ and $1.11(0.65$ to $1.92 ; \mathrm{P}=0.697)$, respectively $(\mathrm{P}$ for interaction $=0.016)$, and for receiving and not receiving statins were $0.93(0.47$ to $1.85 ; \mathrm{P}=0.842)$ and $2.50(1.71$ to $3.64 ; \mathrm{P}<0.001)$, respectively $(\mathrm{P}$ for interaction $=0.013)$.

\section{Discussion}

Findings from the present study demonstrated that prior ischemic stroke in patients with HF was associated with a significantly higher risk of poor outcomes. The associations with all-cause death or readmission became significant during the first 30 days and lasted for 1 year. To the best of our knowledge, this is the first study to compare the risks of death and readmission in HF patients with and without a history of ischemic stroke through one-to-one propensity score matching, which suggested that ischemic stroke remains a strong predictor of unfavorable outcomes after eliminating the greater proportion of potential confounding variables. These findings complement and extend knowledge about prognosis in the presence of ischemic stroke among patients with HF.

There is a general perception that the presence of stroke is associated with a higher burden of comorbidity and poor outcomes in patients with HF. However, several large cohorts of HF patients did not identify stroke as a significant predictor of death or readmission in Western populations (Europe and the USA). ${ }^{7-9}$ Whether stroke per se, rather than additional comorbidities or older age, accounts for the adverse prognosis is a great uncertainty. The intrinsic association between stroke and outcomes of HF may be concealed by the bias of higher prevalence of additional comorbidities affecting $\mathrm{HF}$ prognosis; furthermore, the bias cannot be diminished by traditional regression-based multivariable risk adjustment models. ${ }^{10}$ In the present study, the findings were distinct from those of previous studies because of our use of a robust propensity-score matching design, which was likely the best study design to allow us to assess directly the extent to which stroke has an independent association with outcomes in HF. A notable 


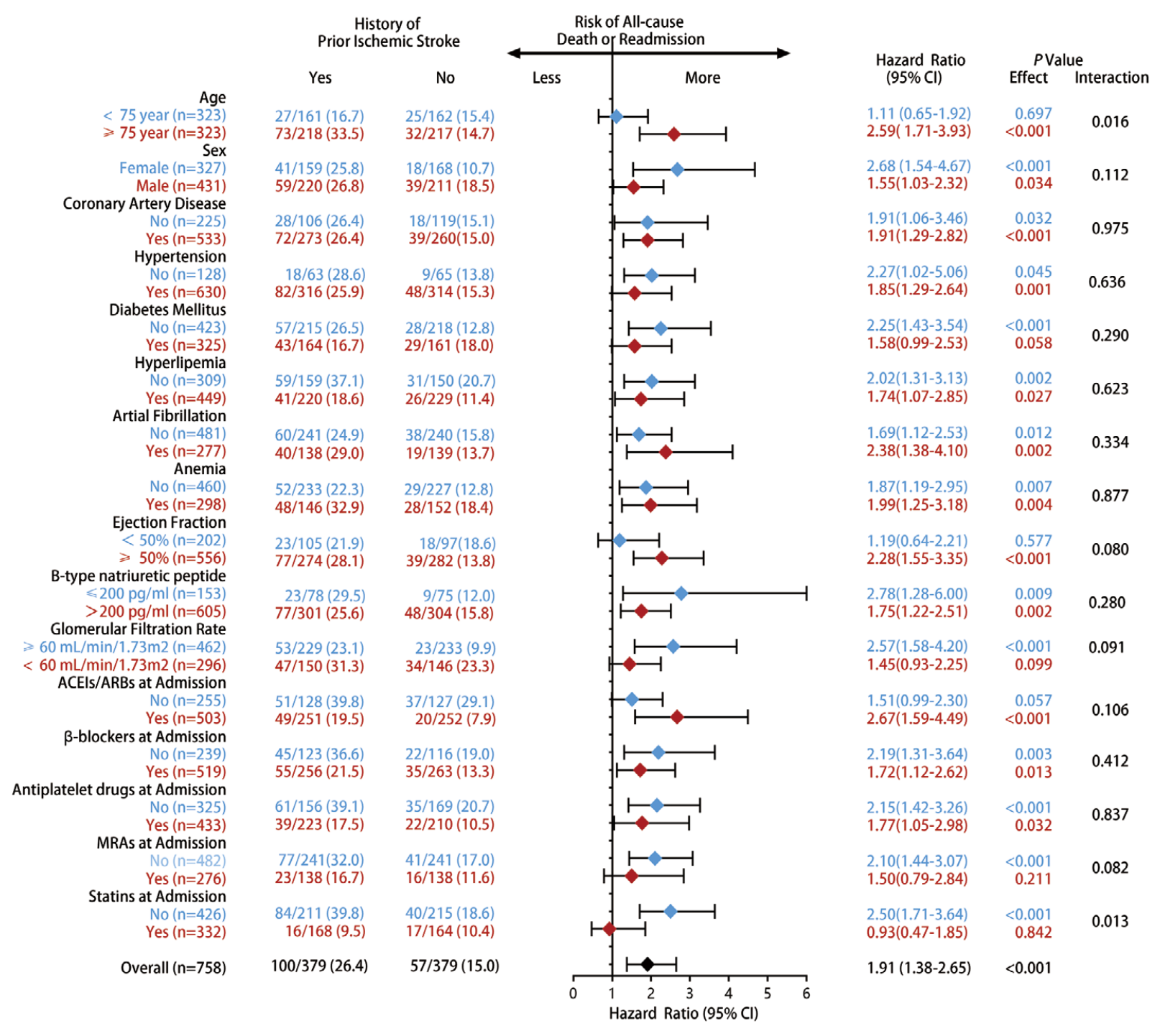

Figure 4. Forest plots for subgroup analysis of all-cause death or readmission, by prior ischemic stroke. ACEI, angiotensinconverting enzyme inhibitor; ARB, angiotensin-receptor blocker; Cl, confidence interval; MRA, mineralocorticoid receptor antagonist.

advantage of propensity score matching is to design observational studies as similar to randomized clinical trials in several critical ways. First, it allowed us to assemble a study cohort in which patients with and without prior ischemic stroke were well balanced on all measured covariates. ${ }^{12}$ Second, we could measure the achieved balance objectively in the matched cohort. ${ }^{11}$ Third, and perhaps most importantly, propensity score matching makes it possible to do both of these procedures without knowledge of, or access to, outcomes data, as investigators of a randomized clinical trial would not know the outcomes of the trial during its design. ${ }^{\mathbf{1 3} 14}$ Therefore, the process of cohort assembly is outcome-blinded as in a randomized clinical trial.

Noteworthy findings of this study were that prior ischemic stroke had a robust effect on all-cause death and readmission in patients with $\mathrm{HF}$, and the effects became significant during the first 30 days and lasted for 1 year.
Ischemic stroke, as an important comorbidity in patients with HF, may promote increasing dysfunction, diminished quality of life and inadequate recovery after HF aggravation. ${ }^{4,6,22}$ Although there is a paucity of studies in both clinical and experimental research investigating the mechanisms of ischemic stroke-induced cardiac dysfunction, several mechanisms are thought to underlie the worsening outcomes of ischemic stroke in patients with HF. Activation of the hypothalamic-pituitary-adrenal (HPA) axis after ischemic stroke causes a significant increase in catecholamines, which can cause cardiac hypertrophy or myocardial ischemia. ${ }^{23-26}$ It is also possible that ischemic stroke could disrupt the blood-brain barrier (BBB) and increased permeability. ${ }^{4}$ In a vicious cycle, disruption of the BBB facilitates the entry of brain-derived antigens and extracellular vesicles derived from injured brain cells to enter the blood stream and encounter peripheral immune cells, which could indirectly induce cardiac dysfunction. ${ }^{27}$ 
Moreover, the complex interplay of the local and systemic inflammatory responses after stroke involves many immune cell types and circulating signals and is important in both infection and secondary cardiac damage., ${ }^{4,2}$ In addition, the role of microvesicles released by damaged astrocytes, neurons and microglia after stroke in mediating cardiac damage warrants future investigation. Thus, HF and prior ischemic stroke are linked by pathophysiological mechanisms that promote the progression of each other, and in turn substantially contribute to poor outcomes in HF patients with prior ischemic stroke.

Prior ischemic stroke has particular relevance for the treatment of HF. HF patients with prior ischemic stroke have complex treatment regimens that can disproportionately lead to polypharmacy and worsen the prognosis of HF patients. However, although prior ischemic stroke has a definite effect on the clinical outcomes of HF, appropriate treatment has not determined in these particular patients with HF. The findings from our subgroup analyses are also important because they help identify the subset of HF patients who are at increased risk of poor outcomes. We observed that the stroke-associated increased risk of death or readmission was higher in those not receiving statins. The evidence regarding the beneficial effects of statin therapy remained contradictory in the general HF population. Currently, the European Society of Cardiology HF guidelines and the American College of Cardiology/American Heart Association HF guidelines do not recommend initiation of statins in most patients with HF. ${ }^{\mathbf{1 6}, 29}$ These recommendations are based mainly on the findings of the CORONA and GISSI-HF studies that were large-scale placebo-controlled trials of statin treatment in patients with HF. ${ }^{30,31}$ No significant decrease in the primary composite mortality/morbidity endpoint was seen in actively treated subjects in either trial. ${ }^{30,31}$ Nonetheless, statins therapy for secondary prevention of ischemic stroke has been recommended by the American Heart Association /American Stroke Association Ischemic Stroke guidelines. ${ }^{32}$ Recently, results from the Treat Stroke to Target trial also suggested intense statin therapy in patients with ischemic stroke of atherosclerotic origin, despite the limitations of the trial. ${ }^{33}$ Statins have multiple cholesterol-independent pleiotropic effects, including the improvement of endothelial function, inhibition of inflammation and thrombosis, promotion of angiogenesis and neuroprotection, and stabilization of atherosclerotic plaques. Pretreatment with a statin is associated with greater neurological improvement and better survival in large artery atherosclerotic stroke. In addition, use of statins may exert a neuroprotective benefit in patients with ischemic stroke through reduction in cholesterol and a number of the pleiotropic effects, which may in turn improve the prognosis of $\mathrm{HF}^{34}$ One of the main protective mechanisms elicited by statin administration is the increase in nitric oxide bioavailability, which regulates cerebral perfusion and improves endothelial function, but others include antioxidant properties, the inhibition of inflammatory responses, immunomodulatory actions, the regulation of progenitor cells, and stabilization of atherosclerotic plaques. Many of these effects are due to the inhibited synthesis of isoprenoid intermediates, which serve as lipid attachments for a variety of intracellular signaling molecules. ${ }^{35}$ Although the data from the present study do not support a change in the existing guidelines and we cannot specifically recommend statin treatment, our results add to evidence that statin therapy has addi- tional prognostic value and may be a treatment option for patients with HF combined with ischemic stroke. To ensure the plausibility and validity of these results, further well-designed controlled trials are needed to fully understand the optimal treatment strategy for this patient population.

Our results identified that the causes of 30-day readmission in patients with $\mathrm{HF}$ and prior ischemic stroke were more likely to be non-HF, for which there are several explanations. First, patients with prior ischemic stroke are prone to dysphagia-related pulmonary aspiration, which is the most common reason for infection, and are admitted into different departments, which may lead to incomplete treatment. ${ }^{36}$ Second, patients with prior ischemic stroke often have unfavorable physical activity levels are thus are prone to physical function impairment, ${ }^{37}$ which can complicate treatment regimens. ${ }^{38,39}$ Third, cognitive impairment after ischemic stroke in many survivors and is a considerable burden to patients and their caregivers, often leading to poor compliance and adherence by the patient. ${ }^{40}$

Our subgroup analyses also found that prior ischemic stroke had a greater effect on HF with preserved ejection fraction (HFpEF) compared with reduced EF (HFrEF). The pathophysiology underlying HFpEF is heterogeneous and no treatment has yet been shown, convincingly, to reduce morbidity or mortality. Therefore, HFpEF patients should be managed with interventions that have been shown to improve symptoms and outcome related to comorbidities. However, our results showed that the treatment of prior ischemic stroke in HFpEF patients was inadequate. First, the use of statins was lower in HFpEF compared with HFrEF patients. Statins may control hyperlipidemia and have pleiotropic effects such as improving vascular endothelial function, inhibition of oxidative stress pathways, and anti-inflammatory actions. 41,42 Accumulating clinical evidence suggests that statins also have a beneficial effect on enhanced cerebral repair in patients with ischemic stroke. ${ }^{43}$ Second, fewer patients with HFpEF appeared to receive $\beta$-blockers compared with patients with HFrEF. Ischemic stroke is a condition associated with a robust stress response with sympathetic activation, which is in turn responsible for increased morbidity and mortality after stroke. ${ }^{\mathbf{4 4 , 4 5}}$ Betablocker therapy has been suggested to have neuroprotective properties, through reducing the increased sympathetic tone after ischemic stroke. ${ }^{46}$

\section{Study Limitations}

Several limitations need to be considered. Given the observational nature of the study, despite a rigorous propensity score matched design to assemble cohorts that were well balanced on all measured baseline covariates, hidden bias may remain because of the influence of potential unmeasured confounders. The conditions associated with stroke, such as disability, cognitive impairment, and frailty, could potentially influence the outcomes of patients with $\mathrm{HF}$, and unfortunately, these conditions were not adequately assessed in our study. Results of subgroup analyses should be interpreted with caution because they may be false positive due to multiple comparisons and false negative due to inadequate power. 


\section{Conclusions}

After accounting for clinical characteristics, prior ischemic stroke was a potent and persistent risk predictor of death and readmission among patients with HF. Given the complex pattern of ischemic stroke and $\mathrm{HF}$, innovative models of integrated multidisciplinary care and further studies on optimal management are warranted specifically in this patient population.

\section{Ethics Approval}

The study was approved by the Ethics Committee of China-Japan Friendship Hospital (No. 2019-83-K54).

\section{Source of Funding}

This work was supported by the National Natural Science Foundation of China (81770359 and 81270276), Central Health Research Project of China (W2017BJ30), and State Key Laboratory of Molecular Developmental Biology of China (2017-MDB-KF-13) to Jingyi Ren.

\section{Disclosures}

The authors declare no conflicts of interest.

\section{Acknowledgments}

None.

\section{References}

1. Benjamin EJ, Muntner P, Alonso A, Bittencourt MS, Callaway CW, Carson AP, et al. Heart disease and stroke statistics - 2019 update: A report from the American Heart Association. Circulation 2019; 139: e56-e528.

2. Tromp J, Tay WT, Ouwerkerk W, Teng THK, Yap J, MacDonald MR, et al. Multimorbidity in patients with heart failure from 11 Asian regions: A prospective cohort study using the ASIAN-HF registry. PLoS Med 2018; 15: e1002541.

3. Lawson CA, Solis-Trapala I, Dahlstrom U, Mamas M, Jaarsma T, Kadam UT, et al. Comorbidity health pathways in heart failure patients: A sequences-of-regressions analysis using cross-sectional data from 10,575 patients in the Swedish Heart Failure Registry. PLoS Med 2018; 15: e1002540.

4. Chen Z, Venkat P, Seyfried D, Chopp M, Yan T, Chen J. Brainheart interaction: Cardiac complications after stroke. Circ Res 2017; 121: 451-468.

5. GBD 2016 Stroke Collaborators. Global, regional, and national burden of stroke, 1990-2016: A systematic analysis for the Global Burden of Disease Study 2016. Lancet Neurol 2019; 18: 439-458.

6. Doehner W, Ural D, Haeusler KG, Čelutkienè J, Bestetti R, Cavusoglu Y, et al. Heart and brain interaction in patients with heart failure: Overview and proposal for a taxonomy. A position paper from the Study Group on Heart and Brain Interaction of the Heart Failure Association. Eur J Heart Fail 2018; 20: 199-215.

7. Dharmarajan K, Hsieh AF, Lin Z, Bueno H, Ross JS, Horwitz LI, et al. Diagnoses and timing of 30-day readmissions after hospitalization for heart failure, acute myocardial infarction, or pneumonia. JAMA 2013; 309: 355-363.

8. Blecker S, Herrin J, Li L, Yu H, Grady JN, Horwitz LI. Trends in hospital readmission of medicare-covered patients with heart failure. J Am Coll Cardiol 2019; 73: 1004-1012.

9. Formiga F, Masip J, Chivite D, Corbella X. Applicability of the heart failure Readmission Risk score: A first European study. Int J Cardiol 2017; 236: 304-309.

10. Fitzmaurice G. Confounding: Regression adjustment. Nutr Burbank Los Angel Cty Calif 2006; 22: 581-583.

11. Rosenbaum PR, Rubin DB. The central role of the propensity score in observational studies for causal effects. Biometrika 1983; 70: $41-55$.

12. Rubin DB. Using propensity scores to help design observational studies: Application to the tobacco litigation. Health Serv Outcomes Res Methodol 2001; 2: 169-188.

13. Deedwania PC, Ahmed MI, Feller MA, Aban IB, Love TE, Pitt
B, et al. Impact of diabetes mellitus on outcomes in patients with acute myocardial infarction and systolic heart failure. Eur $J$ Heart Fail 2011; 13: 551-559.

14. Malik A, Masson R, Singh S, Wu WC, Packer M, Pitt B, et al. Digoxin discontinuation and outcomes in patients with heart failure with reduced ejection fraction. J Am Coll Cardiol 2019; 74: $617-627$.

15. Chinese Society of Cardiology of Chinese Medical Association, Editorial Board of Chinese Journal of Cardiology. Chinese guidelines for the diagnosis and treatment of heart failure 2014. Zhonghua Xin Xue Guan Bing Za Zhi 2014; 42: 98-122.

16. Ponikowski P, Voors AA, Anker SD, Bueno H, Cleland JGF, Coats AJS, et al. 2016 ESC guidelines for the diagnosis and treatment of acute and chronic heart failure. Eur J Heart Fail 2016; 18: $891-975$.

17. American Heart Association Stroke Council, Council on Cardiovascular and Stroke Nursing, Council on Clinical Cardiology, and Council on Peripheral Vascular Disease. Guidelines for the prevention of stroke in patients with stroke and transient ischemic attack. Stroke 2014; 45: 2160-2236.

18. Ahmed A, Fonarow GC, Zhang Y, Sanders PW, Allman RM, Arnett DK, et al. Renin-angiotensin inhibition in systolic heart failure and chronic kidney disease. Am J Med 2012; 125: 399410.

19. Ahmed A, Husain A, Love TE, Gambassi G, Dell'Italia LJ, Francis GS, et al. Heart failure, chronic diuretic use, and increase in mortality and hospitalization: An observational study using propensity score methods. Eur Heart J 2006; 27: 1431 -1439.

20. Ahmed A, Rich MW, Zile M, Sanders PW, Patel K, Zhang Y, et al. Renin-angiotensin inhibition in diastolic heart failure and chronic kidney disease. Am J Med 2013; 126: 150-161.

21. Austin PC. An introduction to propensity score methods for reducing the effects of confounding in observational studies. Multivar Behav Res 2011; 46: 399-424.

22. Haeusler KG, Laufs U, Endres M. Chronic heart failure and ischemic stroke. Stroke 2011; 42: 2977-2982.

23. Barber M, Morton JJ, Macfarlane PW, Barlow N, Roditi G, Stott DJ. Elevated troponin levels are associated with sympathoadrenal activation in acute ischaemic stroke. Cerebrovasc Dis Basel Switz 2007; 23: 260-266.

24. Sykora M, Diedler J, Turcani P, Hacke W, Steiner T. Baroreflex: A new therapeutic target in human stroke? Stroke 2009; 40: e678-e682.

25. Schömig A. Catecholamines in myocardial ischemia: Systemic and cardiac release. Circulation 1990; 82: II13-II22.

26. Samuels MA. Neurogenic heart disease: A unifying hypothesis. Am J Cardiol 1987; 60: 15J-19J.

27. Varatharaj A, Galea I. The blood-brain barrier in systemic inflammation. Brain Behav Immun 2017; 60: 1-12.

28. Vahidy FS, Parsha KN, Rahbar MH, Lee M, Bui TT, Nguyen $\mathrm{C}$, et al. Acute splenic responses in patients with ischemic stroke and intracerebral hemorrhage. J Cereb Blood Flow Metab 2016; 36: $1012-1021$.

29. Yancy CW, Jessup M, Bozkurt B, Butler J, Casey DE Jr, Colvin MM, et al. 2017 ACC/AHA/HFSA focused update of the 2013 ACCF/AHA guideline for the management of heart failure: A report of the American College of Cardiology/American Heart Association Task Force On Clinical Practice Guidelines and the Heart Failure Society of America. Circulation 2017; 136: e137e161.

30. Kjekshus J, Apetrei E, Barrios V, Böhm M, Cleland JGF, Cornel $\mathrm{JH}$, et al. Rosuvastatin in older patients with systolic heart failure. N Engl J Med 2007; 357: 2248-2261.

31. GISSI-HF investigators. Effect of rosuvastatin in patients with chronic heart failure (the GISSI-HF trial): A randomised, double-blind, placebo-controlled trial. Lancet 2008; 372: 1231 1239.

32. Powers WJ, Rabinstein AA, Ackerson T, Adeoye OM, Bambakidis NC, Becker K, et al. Guidelines for the early management of patients with acute ischemic stroke: 2019 update to the 2018 guidelines for the early management of acute ischemic stroke: A guideline for healthcare professionals from the American Heart Association/American Stroke Association. Stroke 2019; 50: $\mathrm{e} 344-\mathrm{e} 418$.

33. Amarenco P, Kim JS, Labreuche J, Charles H, Abtan J, Béjot Y, et al. A comparison of two LDL cholesterol targets after ischemic stroke. N Engl J Med 2020; 382: 9-19.

34. Ní Chróinín D, Asplund K, Åsberg S, Callaly E, CuadradoGodia E, Díez-Tejedor E, et al. Statin therapy and outcome after ischemic stroke: Systematic review and meta-analysis of observa- 
tional studies and randomized trials. Stroke 2013; 44: 448-456.

35. Cimino M, Gelosa P, Gianella A, Nobili E, Tremoli E, Sironi L. Statins: Multiple mechanisms of action in the ischemic brain. Neuroscientist 2007; 13: $208-213$.

36. Lin HJ, Chang WL, Tseng MC. Readmission after stroke in a hospital-based registry: Risk, etiologies, and risk factors. Neurology 2011; 76: 438-443

37. McHutchison CA, Cvoro V, Makin S, Chappell FM, Shuler K, Wardlaw JM. Functional, cognitive and physical outcomes 3 years after minor lacunar or cortical ischaemic stroke. J Neurol Neurosurg Psychiatry 2019; 90: 436-443.

38. Damluji AA, Forman DE, van Diepen S, Alexander KP, Page RL 2nd, Hummel SL, et al. Older adults in the cardiac intensive care unit: Factoring geriatric syndromes in the management, prognosis, and process of care. A scientific statement from the American Heart Association. Circulation 2020; 141: e6-e32.

39. Miró Ò, García Sarasola A, Fuenzalida C, Calderón S, Jacob J, Aguirre A, et al. Departments involved during the first episode of acute heart failure and subsequent emergency department revisits and rehospitalisations: An outlook through the NOVICA cohort. Eur J Heart Fail 2019; 21: 1231 - 1244.

40. Baker DJ, Petersen RC. Cellular senescence in brain aging and neurodegenerative diseases: Evidence and perspectives. J Clin Invest 2018; 128: $1208-1216$

41. Tousoulis D, Oikonomou E, Siasos G, Stefanadis C. Statins in heart failure: With preserved and reduced ejection fraction. An update. Pharmacol Ther 2014; 141: 79-91.

42. van Dongen MME, Aarnio K, Martinez-Majander N, Majander $\mathrm{N}$, Pirinen J, Sinisalo J, et al. Use of statins after ischemic stroke in young adults and its association with long-term outcome. Stroke 2019; 50: 3385-3392.

43. Ní Chróinín D, Asplund K, Åsberg S, Callaly E, CuadradoGodia E, Díez-Tejedor E, et al. Statin therapy and outcome after ischemic stroke: Systematic review and meta-analysis of observational studies and randomized trials. Stroke 2013; 44: 448-456.

44. Sykora M, Diedler J, Rupp A, Turcani P, Rocco A, Steiner T. Impaired baroreflex sensitivity predicts outcome of acute intracerebral hemorrhage. Crit Care Med 2008; 36: 3074-3079.

45. Colivicchi F, Bassi A, Santini M, Caltagirone C. Prognostic implications of right-sided insular damage, cardiac autonomic derangement, and arrhythmias after acute ischemic stroke. Stroke 2005; 36: 1710-1715.

46. Sykora M, Siarnik P, Diedler J; VISTA Acute Collaborators. $\beta$-Blockers, pneumonia, and outcome after ischemic stroke: Evidence from virtual international stroke trials archive. Stroke 2015; 46: $1269-1274$

\section{Supplementary Files}

Please find supplementary file(s); http://dx.doi.org/10.1253/circj.CJ-20-0210 